\title{
ESTUDO ANTROPOMÉTRICO DE PRÉ-ESCOLARES DA CALHA DO RIO AMAZONAS, BRASIL. I - NHAMUNDÁ.
}

\author{
Fernando Hélio ALENCAR, Lúcia Kiyoko O. YUYAMA, Dionísia \\ NAGAHAMA, Tatiana Melo LOPES.
}

\begin{abstract}
RESUMO - Foi realizado no município de Nhamundá (1998) estudo transversal, onde avaliouse o estado nutricional de 152 pré- escolares da área urbana e 30 da área rural, por meio de avaliação antropométrica, adotando-se a classificação de Gomez (1946) e os critérios propostos pela OMS (1986). Constatou-se pela classificação de Gomez que 52,6\% das crianças da área urbana apresentavam algum grau de desnutrição, predominando a forma leve (DI), 9,2\% na forma moderada (DII). Na área rural o quadro se manifesta mais precário, tanto em ocorrência da desnutrição $(66,6 \%)$, quanto na sua manifestação de gravidade (DIII) $3,3 \%$. Segundo os critérios da OMS, $26,9 \%$ das crianças do município apresentavam inadequação no indicador "altura/idade" (desnutrição crônica), constatando-se menor comprometimento das crianças no primeiro ano de vida $(4,8 \%)$. A inadequação no indicador "peso/altura" (desnutrição aguda), foi diagnosticada em $3,3 \%$ dos pré-escolares.
\end{abstract}

Palavras-chave: antropometria; estado nutricional; desnutrição aguda; nanismo nutricional.

Anthropometric Study of Pre-school Children in the Amazon Basin of Brazil. I-Nhamundá.

\begin{abstract}
The nutritional state of 152 urban and 30 rural pre-school children was evaluated on antropometric criteria, using the 1946 Gomez and 1986 WHO classifications, in a transverse study in Nhamundá municipal district, Amazonas State, Brazil, during 1998. In the Gomez classification $52,75 \%$ of the children in the urban area presented some degree of malnutrition, with the light form (DI) predominating, but with a prevalence of $9,2 \%$ the moderate form (DII). In the rural of area the situation was more precarious, both in terms of total prevalence $(66,6 \%)$ and severity of manifestation, with $(3,3 \%)$ of the sample classified as DIII. According to the WHO criteria $26,9 \%$ this sample presente substandard scores of height for age (indicative of chronic malnutrition), less evident in the first year of life $(4,8 \%)$. Acute malnutrition indicated by low weight for height was diagnosed in $3,3 \%$ of these children.
\end{abstract}

Key-words: Nutritional status; anthropometry; acute malnutrition; nutritional stunting.

\section{INTRODUÇÃO}

O perfil mundial da fome estimado pelo UNICEF (1998) é de uma prevalência de 226 milhões de crianças menores de 5 anos com nanismo nutricional, contribuindo o Brasil com $16 \%$ na composição deste quadro epidemiológico entre os países da América Latina. Não obstante, há evidências demonstrando que vem se iniciando, nestes últimos anos, uma melhoria na qualidade de vida da população infantil dos paises em desenvolvimento, notadamente, no que se refere a análise evolutiva dos principais problemas de saúde infantil: pneumonia, diarréia, sarampo, tétano, coqueluche e desnutrição. Registra-se também uma expectativa otimista de erradicação da deficiência de iodo, "que é responsável pelo cretinismo de 120 mil recém-nascidos a cada ano, e a principal causa do retardamento mental de crianças em todo o mundo". Do mesmo modo, a deficiência de vitamina A, principal causa de morte prematura de menores de 5 anos em 
todo o mundo, também, vislumbra-se sua erradicação" (UNICEF, 1995). Entretanto este horizonte de otimismo ainda não pode ser constatado para todo o território nacional. Na Amazônia Brasileira, estudo das condições de vida, saúde e nutrição, abrangendo os diferentes segmentos e grupos populacionais, são relativamente escassos, alguns dos quais realizados, há praticamente duas décadas. Mais freqüentemente, estas pesquisas se referem, principalmente, a população infantil de Manaus, sendo raros e fragmentados estudos enfocando as condições de saúde, nutrição, sobrevivência, e, qualidade de vida das populações do interior da Amazônia. Não obstante, os dados referidos nestes trabalhos, ainda representam parâmetros importantes ao conhecimento e entendimento do processo saúde/ doença, e, carências nutricionais que afetam as populações da região Norte do Brasil (Marinho et al., 1989; Yuyama et al., 1989; Alencar et al., 1991).

A análise evolutiva dos trabalhos realizados no Amazonas nestas duas últimas décadas, mostra como diagnóstico inicial, um perfil pluricarencial em alguns grupos populacionais, caracterizado pela deficiência de micro e macro nutrientes e processos infecciosos, tais como: Baixa ingestão de zinco, vitamina-A, tiamina, riboflavina, cálcio e calorias em estudo alimentar procedido em 1.200 famílias residentes na zona urbana de Manaus (Shrimpton \& Giugliano, 1979). Elevado índice de déficit pôndero-estrutural, alta freqüencia de parasitos gastroentestinais, baixo peso ao nascer e de desmame precoce em pré-escolares e lactentes da área urbana de Manaus (Giugliano et al,1976; Giugliano et al,1981; Giugliano et al,1984; Lehti,1989). Alta freqüência de sinais clinicos de deficiência de vitamina-A, riboflavina, anemia, cárie dental, déficit ponderal, alta percentagem de niveis de hemoglobina abaixo de $11 \mathrm{mg} / \mathrm{dL}$, em pré-escolares residentes na área urbana e rural da Amazônia (Lovenstein,1967; Giuliano et al., 1981, 1984; Nagahama et al., 1990; Marinho et al., 1992). A persistência desta caótica situação alimentar, justifica plenamente a conclusão da Pesquisa Nacional sobre Saúde e Nutrição - (INAN, 1990), que dimensionou para a população infantil da região Norte, um dos maiores déficits estaturais quando comparado com a realidade nutricional infantil das demais macroregiões do Brasil. Neste sentido, o presente estudo objetivou a identificação do estado nutricional dos pré-escolares do municipio de Nhamundá. O somatório destas informações com os resultados oriundos de outras pesquisas que estão em desenvolvimento pelo grupo da nutrição da CPCS/INPA, viabilizará a composição do diagnóstico e mapeamento dos processos carenciais das populações dos diferentes ecossistemas Amazônicos: Rio Negro, Amazonas, Solimões, Madeira , Purus, entre outros.

\section{CASUÍSTICA E MÉTODOS Área Geográfica}

Nhamundá é o segmento municipal, mais distal da Calha do Rio 
Amazonas, em relação a capital do Estado. Dista de Manaus $375 \mathrm{~km}$ em linha reta, e $577 \mathrm{~km}$ por via fluvial. Tem uma área de $14.040 \mathrm{~km}(60 \%$ de terras firmes), o que representa aproximadamente $1,0 \%$ da área total do Estado. Com uma população de 20.172 habitantes (1995), configurando assim uma densidade demográfica de 1,4 hab/ $\mathrm{km}$. Localiza-se à margem direita do rio Amazonas a uma altitude de $50 \mathrm{~m}$ acima do nível do mar. Situa-se a $2^{\circ} 13^{\prime} 25^{\prime \prime}$ de latitude sul e $56^{\circ} 44^{\prime} 21^{\prime \prime}$ de longitude a Oeste de Greenwich, limitando-se com o Município de Parintins, Urucará, e, com os estados de Roraima e Pará (SEBRAE-AM/1994,1995).

\section{População}

O presente estudo envolveu as crianças cujos pais em 1998 residiam no município de Nhamundá há pelo menos 2 anos. $\mathrm{O}$ universo estudado foi identificado por cadastramento populacional (busca ativa), realizado em todos os bairros, e, em uma localidade rural próxima de Nhamundá. Foram incluídas no presente estudo, exclusivamente, as famílias que apresentavam crianças em idade igual ou inferior a 5 anos. Obedecendo estes critérios o universo estudado ficou representado por 182 pré-escolares, sendo 152 residentes na área urbana e 30 na área rural.

\section{Avaliação do Estado Nutricional}

As variáveis peso, altura e comprimento foram obtidas de acordo com as recomendações de Jelliffe (1968). Avaliou-se o estado nutricional dos pré-escolares, empregando-se, inicialmente, a classificação de Gomez
(1946), que utiliza o indicador "peso/ idade" em função do sexo e classifica o estado nutricional em percentagem de adequação em relação a população de referência, adotando-se os seguintes pontos de corte: Normal (> 90\%); desnutridos do grau I ( $90 \%$ a $75 \%$ ); grau II $(75 \%$ a $60 \%)$ e grau III $(<60 \%)$. Na diferenciação dos tipos de desnutrição, utilizamos os critérios propostos pela OMS (1986), que considera como limite discriminatório entre desnutrição/ eutrofia, desvios correspondentes a população de referência (NCHS-1977) inferior a " $<-2$ Escores Z", e, percentil $\leq 10$ para os indicadores: "altura/idade" (desnutrição pregressa e/ou crônica), "peso/altura" (desnutrição aguda) e "peso/idade" (desnutrição global). O "escores z", é igual ao número de unidades de desvios-padrão entre a medida observada e o seu respectivo valor esperado para a população de referência, e o percentil identifica a posição hierárquica que uma determinada variável ocupa dentro de um conjunto de dados.

\section{Análise Estatística}

Para análise dos dados foram utilizados os testes de comparação de duas proporções e o qui-quadrado de Pearson, com nível de significância de $5 \%$, obtidas pelo programa Measure/ Epi-Info-6.0.

\section{RESULTADOS}

A Tabela 1 mostra as medidas de prevalências da desnutrição para os diferentes critérios de avaliação nutricional. Segundo a classificação de Gomez, 54,9\% dos pré-escolares do 
município de Nhamundá, apresentavam algum grau de desnutrição: $46,7 \%$ na forma leve (DI), 7,7\% na moderada (DII) e $0,5 \%$ na forma grave (DIII). Na área rural a desnutrição acometeu $66,7 \%$ das crianças, predominando a forma leve $(63,4 \%)$, entretanto, registrando-se uma ocorrência de 3,3\% de crianças com desnutrição grave. Pelos critérios da OMS, observa-se que a inadequação no indicador peso/idade atingiu $15,4 \%$ dos pré-escolares, a forma crônica $26,9 \%$,e, a aguda acometeu 3,8\% do universo estudado. A distribuição dos resultados em percentís evidencia que $51,1 \%$ dos préescolares se situavam num nivel compatível com desnutrição crônica.

As crianças da área urbana, no primeiro ano de vida, apresentaram uma relativa proteção a sofrer desnutrição, principalmente, na forma crônica $(4,8 \%)$; sendo nula a ocorrência desta forma de desnutrição na área rural. Em ambas as localidades, a ocorrência de desnutrição crônica foi mais freqüente no sexo masculino, entretanto esta contingência não teve expressão estatisticamente significativa $(p<0,05)$. (Tabs 2 e 3 )

\section{DISCUSSÃO}

Apesar das evidências atuais, demonstrando expressiva melhora do estado nutricional das crianças brasileiras, a Desnutrição Energético Proteica (DEP), ainda representa o principal problema de saúde para a população infantil do Norte e Nordeste, ocorrendo graves conseqüências para o seu crescimento físico e desenvolvimento mental,

Tabela 1. Prevalência da Desnutrição Proteico-Energético em Pré-escolares, segundo alguns critérios da Avaliação Nutricional. Município de Nhamundá (Rio Amazonas/1998).

\begin{tabular}{|c|c|c|c|c|c|c|}
\hline \multirow{3}{*}{$\begin{array}{c}\text { INDICADOR } \\
\text { ANTROPOMÉTRICO }\end{array}$} & \multicolumn{6}{|c|}{ ÁREA ESTUDADA } \\
\hline & \multicolumn{2}{|c|}{ URBANA } & \multicolumn{2}{|c|}{ RURAL } & \multicolumn{2}{|c|}{ MUNICIPIO } \\
\hline & $n$ & $\%$ & $n$ & $\%$ & $n$ & $\%$ \\
\hline \multicolumn{7}{|l|}{ GOMEZ } \\
\hline DI & 66 & 43,4 & 19 & 63,4 & 85 & 46,7 \\
\hline DII & 14 & 9,2 & - & - & 14 & 7,7 \\
\hline DIII & - & - & 01 & 3,3 & 01 & 0,5 \\
\hline \multicolumn{7}{|l|}{ OMS (<-2 Scores Z) } \\
\hline Altura/ldade & 42 & 27,6 & 07 & 23,3 & 49 & 26,9 \\
\hline Peso/Altura & 05 & 3,3 & 02 & 6,7 & 07 & 3,8 \\
\hline Peso/ldade & 23 & 15,1 & 05 & 16,7 & 28 & 15,4 \\
\hline \multicolumn{7}{|l|}{ PERCENTIL } \\
\hline Altura/ldade & 75 & 49,3 & 18 & 60,0 & 93 & 51,1 \\
\hline Peso/Altura & 15 & 9,9 & 04 & 13,3 & 19 & 10,4 \\
\hline Peso/ldade & 62 & 40,8 & 08 & 26,7 & 70 & 38,5 \\
\hline Crianças estudadas & 152 & $(100,0)$ & 30 & $(100,0)$ & 182 & $(100,0)$ \\
\hline
\end{tabular}

$\mathrm{n}=$ número de crianças 
Tabela 2. Situação nutricional de Pré-escolares, da área urbana segundo os critérios da Avaliação da OMS, faixa etária e Sexo. Município de Nhamundá (Rio Amazonas/1998).

\begin{tabular}{|c|c|c|c|c|}
\hline \multirow{2}{*}{$\begin{array}{l}\text { Variáveis } \\
\text { Analisadas }\end{array}$} & \multirow[b]{2}{*}{$n$} & \multicolumn{2}{|c|}{ Critérios de Avaliaçăo } & \multirow[b]{2}{*}{$\begin{array}{l}\text { Peso/Altura } \\
-2 \text { Scores Z }\end{array}$} \\
\hline & & $\begin{array}{l}\text { Peso/ldade } \\
-2 \text { Scores Z }\end{array}$ & $\begin{array}{l}\text { Altura/ldade } \\
-2 \text { Scores } Z\end{array}$ & \\
\hline \multicolumn{5}{|c|}{ IDADE (MESES) } \\
\hline $0-12$ & 27 & $1(4,3 \%)$ & $2(4,8 \%)$ & $2(40,0 \%)$ \\
\hline $13-24$ & 27 & $7(30,4 \%)$ & $8(19,0 \%)$ & $1(20,0 \%)$ \\
\hline $25-36$ & 26 & $4(17,4 \%)$ & $6(14,3 \%)$ & $-(-)$ \\
\hline $37-48$ & 35 & $5(21,7 \%)$ & $11(26,2 \%)$ & $-(-)$ \\
\hline $49-60$ & 37 & $6(26,1 \%)$ & $15(35,7 \%)$ & $2(40,0 \%)$ \\
\hline Total & 152 & $23(15,1 \%)$ & $42(27,6 \%)$ & $5(3,3 \%)$ \\
\hline \multicolumn{5}{|l|}{ SEXO } \\
\hline Masculino & 81 & $10(43,5 \%)$ & $24(57,1 \% 0$ & $3(60,0 \%)$ \\
\hline Feminino & 71 & $13(56,5 \% 0$ & $18(42,9 \%)$ & $2(40,0 \%)$ \\
\hline$c^{2}\left({ }^{*}\right)$ & & 0,3075 & 0,5575 & 0,7605 \\
\hline Total & 152 & $23(110,0 \%)$ & $42(100,0 \%)$ & $5(100,0 \%)$ \\
\hline
\end{tabular}

(*) Mantel-Haenszel

$\mathrm{n}=$ número de crianças

Tabela 3. Situação nutricional de Pré-escolares, da área rural, segundo os critérios da Avaliação da OMS, faixa etária e Sexo. Município de Nhamundá (Rio Amazonas/1998).

\begin{tabular}{|c|c|c|c|c|}
\hline \multirow[t]{2}{*}{$\begin{array}{l}\text { Variáveis } \\
\text { Analisadas }\end{array}$} & \multirow[b]{2}{*}{$n$} & \multicolumn{2}{|c|}{ Critérios de Avaliação } & \multirow[b]{2}{*}{$\begin{array}{l}\text { Peso/Altura } \\
-2 \text { Scores Z } \\
\end{array}$} \\
\hline & & $\begin{array}{l}\text { Peso/ldade } \\
-2 \text { Scores Z }\end{array}$ & $\begin{array}{l}\text { Altura/ldade } \\
-2 \text { Scores } Z\end{array}$ & \\
\hline \multicolumn{5}{|c|}{ IDADE (MESES) } \\
\hline $0-12$ & 3 & $-(-)$ & $-(-)$ & $-(-)$ \\
\hline $13-24$ & 8 & $2(40,0 \%)$ & $2(28,6 \%)$ & $2(100,0 \%)$ \\
\hline $25-36$ & 9 & $2(40,0 \%)$ & $3(42,9 \%)$ & $-(-)$ \\
\hline $37-48$ & 6 & $1(20,0 \%)$ & $1(14,3 \%)$ & $-(-)$ \\
\hline $49-60$ & 4 & $-(-)$ & $1(14,3 \%)$ & $-(-)$ \\
\hline Total & 30 & $5(16,7 \%)$ & $7(23,3 \%)$ & $2(6,7 \%)$ \\
\hline \multicolumn{5}{|l|}{ SEXO } \\
\hline Masculino & 18 & $4(80,0 \%)$ & $4(57,1 \% 0$ & $-(-)$ \\
\hline Feminino & 12 & $1(20,0 \%)$ & $3(42,9 \%)$ & $2(100,0 \%)$ \\
\hline$c^{2} \quad(*)$ & & 0,3225 & 0,8624 & 0,0779 \\
\hline Total & 30 & $5(100,0 \%)$ & $7(100,0 \%)$ & $2(100,0 \%)$ \\
\hline
\end{tabular}


sendo ainda responsável pela baixa resistência as doenças e elevado índice de mortalidade infantil (INAN,1990; OMS, 1993; UNCEF, 1994, 1995).

Na investigação da ocorrência de DEP nas crianças de Nhamundá, utilizamos a conjugação de vários critérios diagnósticos. A classificação de Gomez (1946), apesar da sua utilização universal, tem suas limitações em estudos epidemiológicos, visto que em não considerando a altura, pouca informação revela a respeito da dinâmica do processo carencial, inviabilizando assim, a diferenciação dos processos carenciais crônicos dos agudos. A utilização desta metodologia evidenciou que na área urbana de Nhamundá predominou a forma leve de desnutrição $(46,7 \%)$, sendo mínima a ocorrência da forma grave $(0,5 \%)$. Na área rural a situação nutricional é mais precária, tanto em termos percentuais de ocorrência da desnutrição $(66,7 \%)$, quanto na sua gravidade $(3,3 \%)$.

A análise evolutiva dos trabalhos desenvolvidos em pré-escolares na região Amazônica, nestas últimas décadas, revela a persistência de um quadro, epidemiologicamente preocupante no que se refere a qualidade de vida da referida população. Por exemplo, para a área rural não se constatou nenhum progresso no estado nutricional da população infantil nestas duas últimas décadas. $\mathrm{O}$ diagnóstico nutricional registrado para os préescolares do presente estudo é, inquestionavelmente, idêntico aquele referido por Giugliano et al, 1981/1984 em pré-escolares do Rio Solimões e Rio Negro, respectivamente. Observa-se ainda ao longo do período analisado, uma tendência de inflexão na curva de prevalência global da DPE; entretanto, os valores percentuais encontrados para as diversas formas de desnutrição (DI e DII), deixa implícito a precariedade da situação nutricional, dado a rapidez com que as formas leve e moderada, evoluem para um estágio de maior gravidade, notadamente, em populações carentes de programas de intervenção epidemiológica e/ou assistência materno-infantil (Alencar et al,1993; Alencar et al, 1999). Depreende-se ainda da confrontação dos resultados destes estudos, que a situação nutricional dos pré-escolares residentes na capital do estado (Manaus), é relativamente superior aquela registrada no contexto rural amazônico (UNICEF, 1996; Alencar et al, 1999). Entretanto a confrontação percentual dos resultados do presente estudo, com aqueles da Pesquisa Nacional sobre Saúde e Nutrição (INAN, 1990), praticamente, nivela em termos epidemiológicos, a situação nutricional no município de Nhamundá $(54,9 \%)$, com a do Nordeste Rural $(52,7 \%)$, diferindo expressivamente as duas populações, na manifestação da gravidade da desnutrição: 8,2\% e 11,7\% (DII + DIII), respectivamente.

No presente estudo, a gravidade da desnutrição foi interpretada utilizando-se os critérios propostos pela (OMS, 1986). O déficit de estatura/idade, que reflete a cronicidade da privação nutricional, identificando assim a condição de nanismo em crianças de 0 a 5 anos, foi registrado em $26,9 \%$ das crianças do 
Município. Constata-se para a área urbana, uma relativa proteção das crianças mais jovens contraírem a desnutrição, sendo mais acometido o sexo masculino $(57,1 \%)$. Na área rural, verificou-se total proteção das crianças a sofrerem qualquer forma de desnutrição no primeiro ano de vida. Do mesmo modo, a utilização da distribuição dos percentís, evidencia também como principal forma de desnutrição para o município de Nhamundá, a inadequação no indicador altura/idade, com $51,1 \%$ dos pré-escolares se situando no nivel igual e/ou inferior ao Percentil 10, que é o limite discriminatório entre desnutrição/eutrofia. $\mathrm{Na}$ região Amazônica, trabalhos enfocando estas metodologias ainda são raros, apesar das recomendações da OMS (1993). Possivelmente o único estudo expressando a inadequação dos indicadores antropométricos em "escores Z", foi realizado por Alencar et $a l$, (1999), no município de Barcelos. Os referidos autores verificaram que $31,1 \%$ e $4,2 \%$ dos pré-escolares daquele município sofriam desnutrição crônica e aguda respectivamente; portanto uma situação nutricional muito parecida com aquela diagnosticada no presente estudo. Entretanto estes percentuais ainda são expressivamente superiores aqueles registrados pela Pesquisa Nacional sobre Saúde e Nutrição - (INAN, 1990), há mais de uma década para os préescolares da região Norte (Urbana). Estes resultados evidenciam a situação de precariedade assistencial e nutricional a que, historicamente, vem vivenciando a população infantil do contexto rural Amazônico.

\section{AGRADECIMENTOS}

Ao BID/FINEP pela ajuda financeira ao desenvolvimento desta pesquisa.(Processo no 64.96.0746-).

\section{Bibliografia citada}

Alencar, F.H.; Ferraroni, M.J.R.;Lehti, K.K.; Marinho, H.A.; Mota, C.S.; Silva, N.B.; Castro, J.S. 1991. Diagnóstico e perspectivas nutricionais na Região Amazônica. In: Val, A.L.; Figliuolo, R. Feldberg, E. (eds) Bases Cientificas para Estratégias de Preservação e Desenvolvimento da Amazônia: Fatos e Perspectivas. Vol. I:145-154.

Alencar, F.H.; Marinho, H. A.; Nascimento, B. A. do.1993. Estudo das condições (nutricional, econômica e social), de crianças pré-escolares residentes na área urbana de Manaus-AM. 45 Reunião Anual da Sociedade Brasileira para o Programa da Ciência. Sociedade Brasileira para o Progresso da Ciência -SPBC.

Alencar, F. H.; Yuyama, L. K. O; Nagahama, D.; Parente, C. P.1999. Estudo Antropométrico de pré-escolares da Calha do Rio Negro, Amazonas, Brasil. IIBarcelos, Acta Amazonica 29(2):293-302.

Giugliano \& Shrimpton, 1976. Estudo antropométrico e clínico de estado nutricional em um grupo de crianças préescolares de Manaus. Acta Amazonica, 7(3):389-394.

Giugliano, R.; Giugliano, L.; Shrimpton, R. 1981. Estudos nutricionais das populações rurais da Amazônia. I. Várzea do Rio Solimòes. Acta Amazonica, 11(4):773788.

Giugliano, R.; Shrimpton, R.; Marinho, H.A.; Giugliano, I. 1984. Estudos nutricionais das populações rurais da Amazônia. II. Rio Negro. Acta Amazonica, 14(3-4):427449 .

Gomez, F. 1946. Desnutrición. Bol Medico. Hosp. Infant. México, 3:53-51.

Instituto Nacional de Alimentação e Nutrição 
- INAN 1990. Pesquisa Nacional sobre Saúde e Nutrição em 1989. Perfil de crescimento da População brasileira de 05 anos. Brasília DF., 59p.

Jellife, D.B.1968. Evaluación del estado de nutrición de la comunidad; com especial referencia a las regiones en desarrollo. Ginebra:Org. Mundial de la Salud. 291p.

Lehti,K.K.1989. Iron, folic acid and zincintakes andstatus of low socio-economic pregnant and lactating Amazoniam Womem. Eur. J. Clin.Nutr; 43:505-513.

Lowenstein, F.W. 1967. Report on nutrition in 11 brasilian Amazon communities betwen 1955 and 1957. Atas do Simpósio sobre Biota Amazonica, vol.6 (patologia):177184.

Marinho, H.A. 1989. Influência de parasitose intestinal (Ascaris lumbricoides e ou Giardia lambia) sobre os niveis séricos de vitamina-A em crianças recebendo suplementação oral de zinco elou vitaminA. Estudo em pré-escolares de um bairro pobre de Manaus, Amazonas. Dissertação de Mestrado. Universidade do Amazonas, $86 \mathrm{p}$.

Marinho, H.A.; Kazapi, I,M.; Fernandes, H.H.J.M.; Guedes, M.R. 1992. Avaliação do estado nutricional de pré-escolares de classe sócio-econômica média e baixa da cidade de Manaus. Estudo comparativo. Acta Amazonica, 22(3):363-368.

NCHS, 1977. Growth curves children birth 18, united States. Washington: National Center for Health Statistics. DC: U.S. Printing Office (Vital and Health Statistics Series 11, pub. $\mathrm{N}^{0} 78-1650$ ).

Nagahama, D.; Marinho, H.A.; Rocha, Y; Ferraroni, M.J.R.; Silva, N.B.; Gosho, J.S.; Onety, J.A. 1990. Avaliação nutricional e alimentar de pré-escolares de uma creche de Manaus e a influência de entidade no estado nutricional de sua população. Acta Amazonica, 20 (único):119-128.

SEBRAE/AM, 1994. Diagnóstico sócioeconômico e cadastro empresarial de Barcelos. Manaus: Departamento de
Estudo e Pesquisa, 199p.

SEBRAE/AM, 1995. Diagnóstico sócioeconômico e cadastro empresarial de Nhamundá. Manaus: Departamento de Estudo e Pesquisa, 199p

Organizacion Mundial De La Salud - OMS. 1986. Use and interpretation of antropometric indicators of nutritional status. Bull. World Health Organ, 64:929-41.

Organizacion Mundial De La Salud - OMS. 1993. Situacion alimentaria y nutricional de America Latina. In: Conferência Internacional sobre Nutricion. Santiago, Chile.

Shrimpton, R.; Giugliano, R. 1979. Consumo de alimentos e alguns nutrientes em Manaus, Acta Amazonica, 9:117-141.

Situação Mundial da Infância. 1994. Fundo das Nações Unidas para a Infância UNICEF.

Situação Mundial da Infância. 1995. Fundo das Nações Unidas para a Infância UNICEF.

Situação Mundial da Infância. 1996. Fundo das Nações Unidas para a Infância UNICEF.

Situação Mundial da Infância. 1998. Fundo das Nações Unidas para a Infância UNICEF.

Yuyama, L. K. O.; Nagahama, D.; Marinho, H.A; Vannuchi. H. 1989. alimentação e estado nutricional de mães em diferentes estados fisiológico de um bairro pobre de Manaus. Alimentos e Nutrição, São Paulo. 1: $13: 21$, 